\title{
Industrial Intelligent Software Applications of the Overall Equipment Effectiveness (OEE) in Manufacturing
}

\author{
Bernd Otto Hörmann ${ }^{1}$, Dan Bizubac ${ }^{1, *}$ and Marcel Sabin Popa ${ }^{1}$ \\ ${ }^{1}$ Technical University of Cluj-Napoca, Department of Manufacturing Engineering, Muncii Blvd 103- \\ 105, Cluj-Napoca, ROMANIA
}

\begin{abstract}
This paper aims to evaluate the solution behaviour of the "Overall Equipment Effectiveness" to determine the performance in innovative manufacturing environments. OEE literature categorizes six major losses: "Equipment failures and breakdowns", "Setup/Adjustments", "Idling and minor Stops", "Reduced Speed Losses", "Reduced Yields and Quality Defects and Rework". The technical implementation method used by the authors to achieve their scope has been described using a hybrid of software development programs such as: SAP MII, SAP PCo and SAP ME in relation to the theoretical existing principles. The results presented conclude that losses in practice can occur at both high rates and low rates and therefore, an innovative manufacturing concept must be further enhanced. In terms of performance related to equipment utilization, the specific stoppages and downtimes need to be taken in consideration and adjust the OEE results accordingly. The actual situation in a real-life environment has increased in efficiency, but as technology improves, opportunities for further development appear at a high rate.
\end{abstract}

\section{Literature Review}

OEE ("Overall Equipment Effectiveness") has been defined in literature as being the tool used to measure the equipment performance in the manufacturing industry. In order to provide a reliable standard, norms and regulations have been created with the purpose of creating a simple and clear solution. Against all of these efforts, the time and rate efficiency constraint can still create imperfections.

In the OEE implementation, the topic of performance measurement has been deeply addressed. Performance measurement addresses the question "To what extend will the company fulfil their objective?".

In his work, Kaydos [1] has identified the main reasons for which this measurement is necessary:

- Detailed control over the system

- A well-defined set of objectives and responsibilities with the purpose of knowing the responsible for each process

* Corresponding author: danbizubac@yahoo.com 
- Enhanced strategy management in order to communicate to all the levels of the organization and act accordingly to adjust the current state to the desired vision of the organization.

- Technical understanding of the actual business process inside the manufacturing plant.

Nakajima [2] introduced OEE in his work using in his "Total Productivity Maintenance" (TPM) concept. This is strictly focused towards machines and equipment used to measure the overall aggregated result, not the minor detailed outcomes. Huang et al. [3] evaluate the concept of OEE as being highly popular in operations and mainly manufacturing. Moreover, the authors debate on wherever this tool is one of the most innovative quantitative measurement processes compared to the traditional metric that usually evaluate only productivity and utilization.

SEMI (Semiconductor Equipment and Materials International) [4] has continued the work on Nakajima [2] and in the year 2000 a new standard for the "Overall Equipment Efficiency" has been established. Ljundberg [5] in 1988 contradicts the existing theory by stating that in OEE some factors should also be taken in consideration such as lack of materials, labour or even the planned downtimes. De Groote [6] has included these times in his model and calculated the differences between them in order to obtain feasible results for the manufacturing plant. As a result of all these authors, OEE has been lately focusing on all of the existing factors in the industry and takes in consideration all the input materials, the availability of those and the equipment itself and the recipes that lie as a foundation for the production process.

In detail, OEE has been divided in the following major categories:

- For equipment breakdown or failures when there is a reduced productivity with justified reason,

- For equipment that need adjustment or setup between the production of a certain amount of pieces,

- For equipment stoppages when a specific machine is idling or malfunctioning,

- Different operating times between the equipment's design and the actual speed,

- Stabilization time losses which usually occur at start-up,

- Time losses for quality or rework defects.

In literature [2], the first two types have been considered as the availability of the machines, the third and the fourth have been considered the speed losses regarding the efficiency and the final two are linked to the defects. In Nakajima's [2] opinion, multiplying the availability, the efficiency and the quality rate will result in the actual OEE.

Availability has been used to describe in theory the measured time of a particular machine that is producing goods at a normal rate. This has been linked in practice to the time that the machine has been scheduled for producing the desired object. Furthermore, "Availability Loss" is defined using the data collected from the machine and it refers to the time when the machine is not operating. The "Plan Operating Time" is described as the normal shift for the machine not including the scheduled downtimes. The machine operator can further divide the "Availability Loss" depending on actual activity on the production line. The operator can insert "Planned or Unplanned Downtimes" by selecting different codes displayed on the physical screen of the machine. "Planned Downtimes" refer to the breaks that the operator is having during his schedule and "Unplanned Downtimes" are unforeseen events that may cause the stopping of the equipment such as power outlets.

Performance indicates the speed at which the physical machines is producing parts in a daily shift. This is defined as being a quantitative indicator of which takes in consideration the actual amount of parts produced compared to the capacity of the equipment in an ideal environment. By using the performance indicator, the machine is evaluated in terms of producing the ideal quantity in the daily "Machine Operating Time" of the shift. 
The third important aspect that needs to be taken in consideration is Quality. Quality refers to the actual accepted quantity of finished goods produces from the total amount of raw material in the actual machine time shift of the day. This topic can be further elaborated with new and improved sophisticated solutions and error-inspection steps that can present the quality performance in the actual machine. OEE defines the production time of quality accepted pieces of finished goods divided by the shift time.

\section{Chosen Methodology}

An important layer of the methodology is described with the product from SAP named "Plant Connectivity" (PCo). The software is used in this case study as a set of tools that will exchange the data between the OEE system and the actual physical system represented by the equipment and machines. The implementation using this type of software relies on the fact that the equipment sends the data as a source system towards the PCo system, which will be connected to the OEE system. Inside the PCo environment, several conditions and tags will evaluate, using complex software architectures and enhanced innovative concepts, the quality of the data received from the production line.

Summarizing, as one may notice from the concepts presented before:

- Three types of PCo agents (instances) per machine have been developed

$\circ$ One "Agent for Capturing the good Quantities": The quantity aggregation is collected inside this agent,

○ One "Agent for Capturing the scrap Quantities": The quantity aggregation is collected inside this agent,

○ One "Agent for Capturing the Stoppage with corresponding PLC" (Programmable Logical Control) Code.

- The PCo agent (instance) responsible for capturing the stoppages mapped the PLC tags to OEE Modes. The agent has been designed to forward the OEE Mode ID to MII (SAP "Manufacturing Intelligence and Integration" in Column C from the Mode Matrix Sheet presented in Fig.1.)

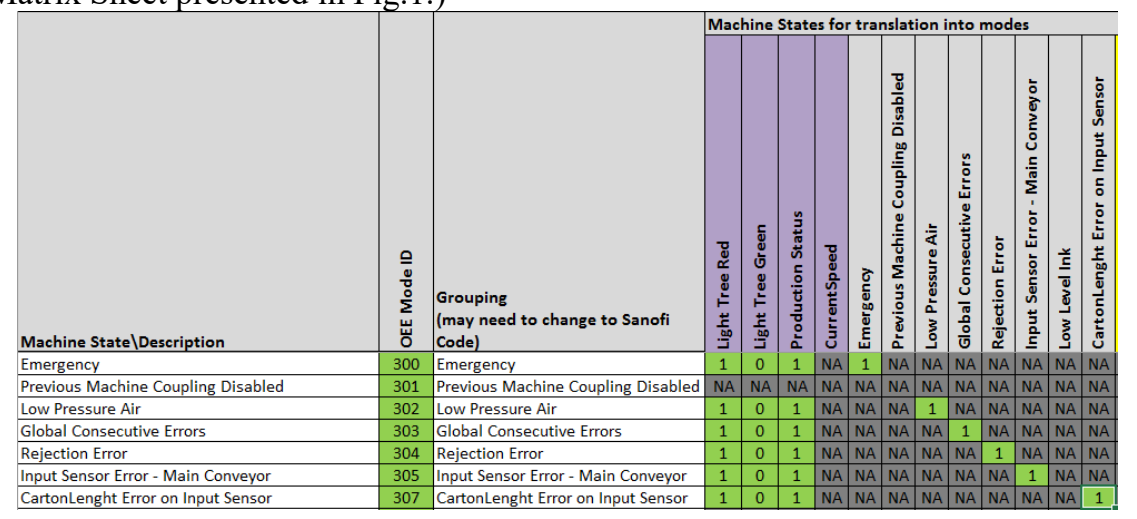

Fig. 1. "Mode Matrix Sheet".

- In SAP MII, the mapping between the machine code, plant hierarchy and reason codes was managed accordingly using programming code. Such a mapping was implemented per line/plant/machine.

- In MII the stoppage events needed to be received. In the case for a new stoppage, MII was responsible for identifying the corresponding SAP OEE reason code based on received OEE Mode, plant and machine ID 
- A pulse has been defined as an information consisting of machine ID, Timestamp and aggregated good or scrap quantity over a period of time,

- A Pco Server dedicated to the plant/line was installed at the plant, in order to meet the requirements,

- $\quad$ SAP MII including SAP OEE became centralised systems serving different plants and lines.

Several assumptions were needed to be made in order to test the scenarios:

$>$ The combination of plant and machine ID has been defined as unique across the entire test landscape,

$>$ The description of the machine code has been defined as not relevant in OEE,

$>$ Stoppages and production quantities (goods or scraps) have been decoupled from each other in terms of data integrity and reliability. The correlation in terms of OEE happened in the standard SAP OEE solution itself,

PLC/OPC server provided "UTC TIMESTAMPS" and the PLC clocked with IT Reference System.

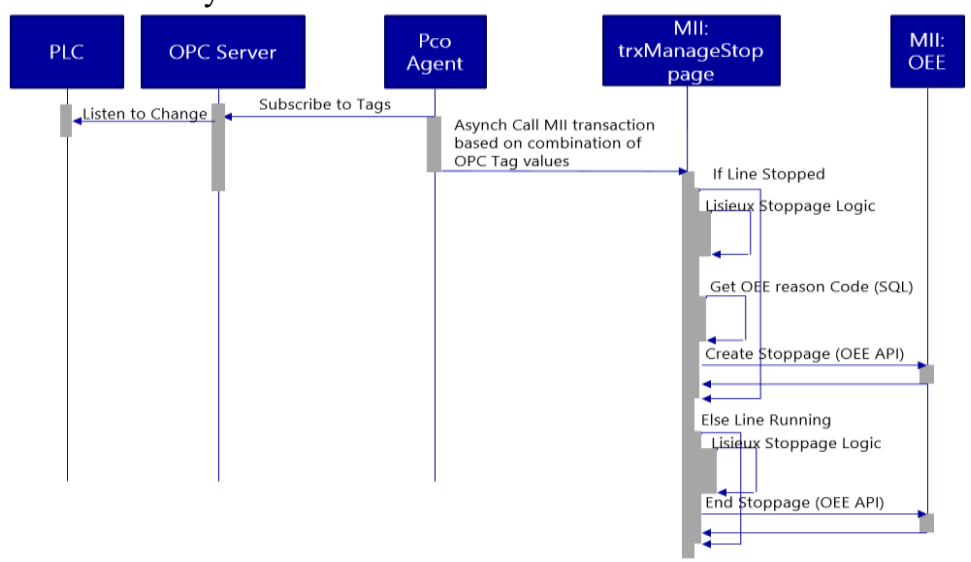

Fig. 2. "Stoppage Management Simplified Sequence" Diagram.

Fig.2. defines the particular process of which the data has been captured. First of all, the PLC (Programmable Logic Controller) captures all the data coming from the machines in a two-digital environment. At this level, the information is only extracted with the purpose of being handled at the next level of the system. The OPC server transforms the information from a two-digital format in a human-readable format. After this conversion, the information has been further transferred to the PCo Agent. The third and one of the core levels of the sequence is being represented by the PCo level. Using different logical conditions, the subscribed information to the PLC Tags is passed based on the OPC values received.

At a "User Interface" level, several tasks are being performed in background. In this process, the researchers have developed a SAP transaction, which is called "trxManageStoppages", which checks if the line is stopped. Then, it will check the generic "Stoppage Logic" and if that is correct, it will further look in the database tables for the respective OEE code linked to the provided reasons codes (please check the reference given in Fig. 1 for the example of the reason codes). If a correct code for the stoppage has been found, then the system will create the stoppage in the software system. Otherwise, the current logic will perform its routine activities as before. For error handling, in the case of a miss match with the OEE code, the software solution will perform its routine activities as before. 


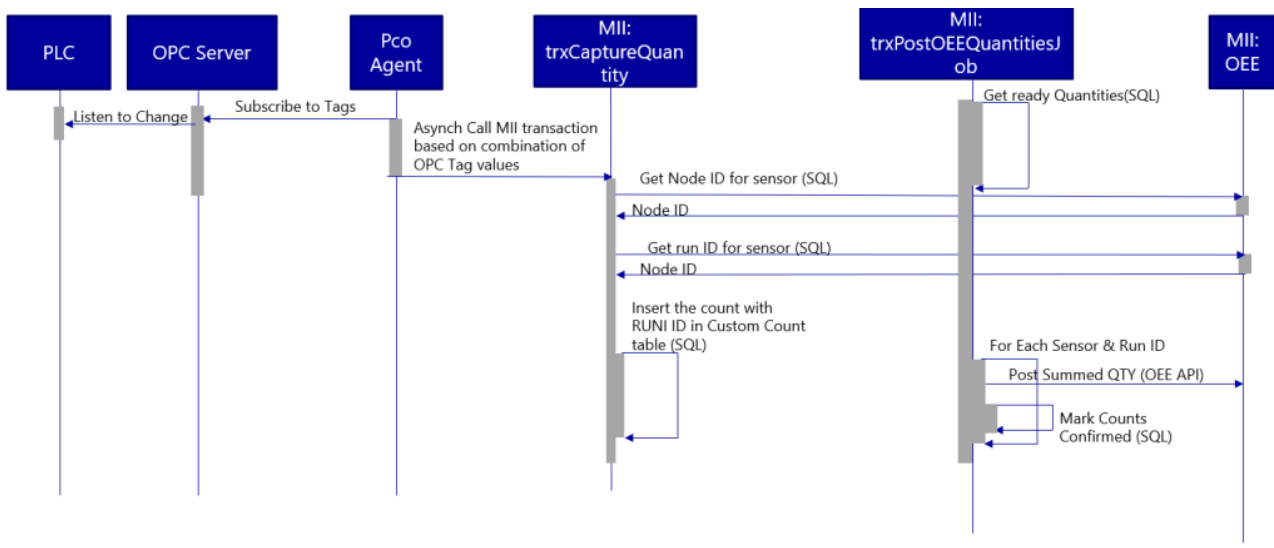

Fig. 3. "Capture Good $\backslash$ Scrap Quantities Simplified Sequence” Diagram.

Fig.3. explains two different other scenarios researched by the authors: the capturing of the good or scrap quantities from the manufacturing machines. The physical sequence is similar to the first case scenario, however, in terms of the transaction and logic used, the actual two scripts use the transactions named: "trxCaptureQuantity" and "trxPostOEEQuantitiesJob". The first transaction runs a query in the database to find the "NodeID" and after the node it has been found, the search in the database is once again started with the purpose of finding the actual sensor. After the sensor has been successfully found, the count with the "RunId" is inserted in the Custom Count Table.

In the second phase of the logic, at the time when the transaction "trxPostOEEQuantitiesJob" is being used, the system searches inside the database to find the actual quantities referred. The programming code performs a sum of all the quantities within and an increment of a counter when a quantity has been found to a particular sensor.

\section{Conclusions}

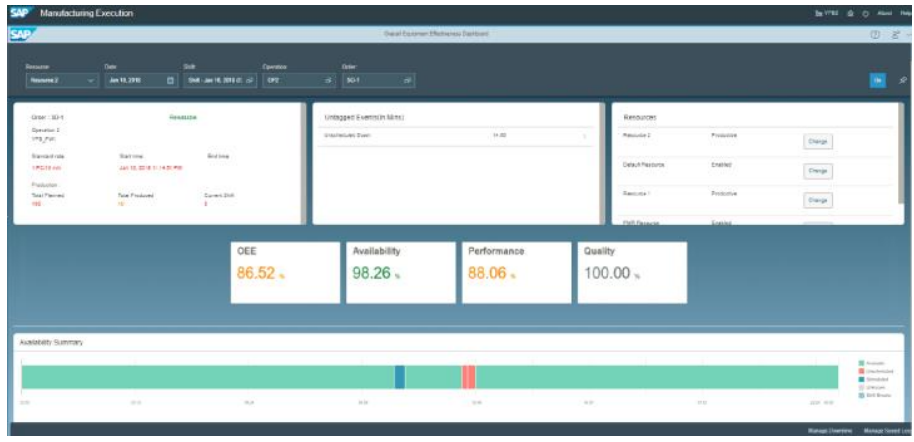

Fig. 4. "Operator Main Dashboard".

In Fig.4. "Operator Main Dashboard", filters for "Resource", "Date", "Shift", "Order" and "Operation" selection can pe applied. In this finding, the authors have displayed also the 3 main categories discussed in literature: "Availability" (98.26\%), "Performance" $(88.06 \%)$ and "Quality" (100\%) for the specified machine resource. 


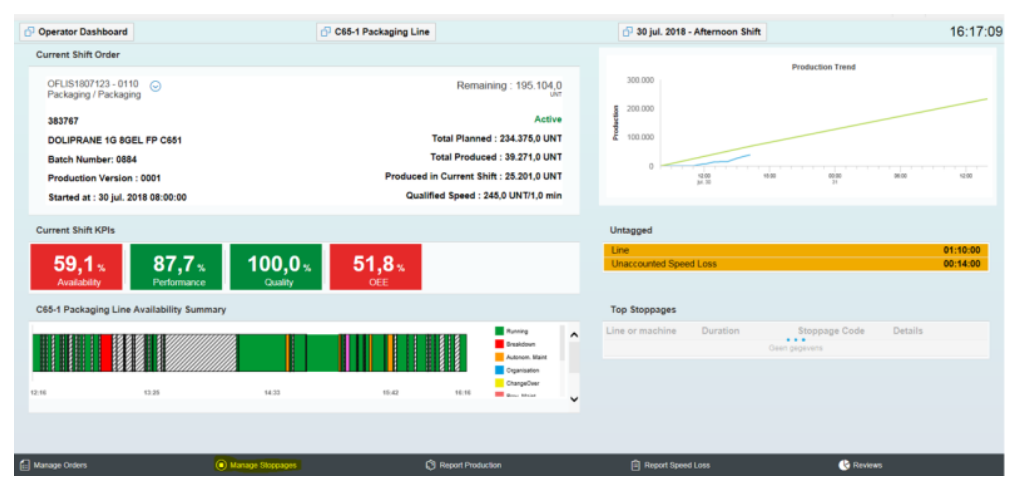

Fig. 5. "Production Line Dashboard" - Operator View.

In Fig.5. "Production Line Dashboard" for packaging, the operator can observe how the equipment is behaving and furthermore, how the machines are reacting in terms of "Availability" (59.1\%), "Performance" (87.7\%) and "Quality" (51.8\%) with stoppages and losses graphic.

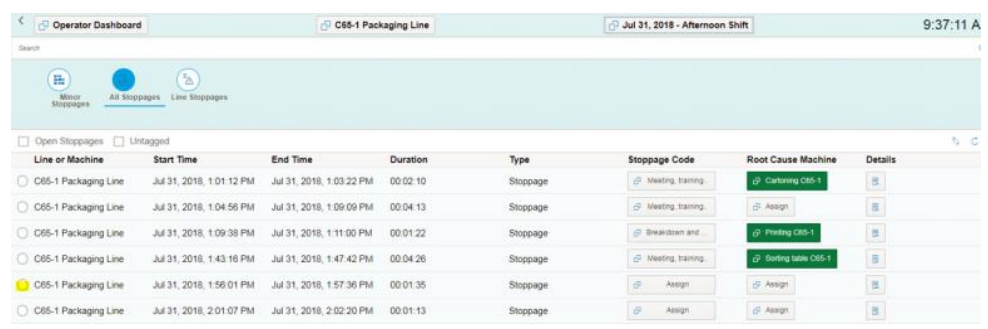

Fig. 6. Personalized Dashboard for the KPI for the Packaging Line where "Reason Code" and "Root Cause" are assigned as stoppages or loses, when needed by the operator.

The reasoning behind OEE software solutions is that a particular manufacturing plant should have a certain metric system that indicates the overall performance. Unfortunately, OEE is not strictly directed to the equipment and the number generates include also the effects caused by other external factors. When evaluating the time losses, the authors have experienced in their work, several other inputs that are not related to the equipment, such as: human breaks, inexistent or not enough buffer time between items or other external environmental factors. The OEE metric has been evaluated by the authors as a successful tool when evaluating strictly the equipment. In the case of evaluating the overall environment, further hypothesizes need to be tested in the future for effective results.

\section{References}

1. Kaydos, W., St. Lucie Press: Boca Raton, FL (1999)

2. Nakajima, S., Productivity Press Inc.: Cambridge, MA (1988)

3. Huang, S.H., Dismukes, J.P., Shi, J., Su, Q., Razzak, M.A., Bodhale, R. and Robinson, D. E., Int. J. Prod. Res., 41, 513-527 (2003)

4. SEMI, E79-0200 (2000)

5. Ljundberg, O., 18, 495-507 (1998)

6. De Groote, P., 1, 4-24 (1995)

7. T. Aschwanden, S. Chakraborty, SAP (2018) 